\title{
Seismic Design of a Out-of-code High-rise Library
}

\author{
Ma Zefeng, Fang Lin \\ East China Architectural Design \& Research Institute Co.,Ltd., Shanghai, China
}

Email address:

mzfmzf@sina.com (Ma Zefeng), lin_fang@arcplus.com.cn (Fang Lin)

\section{To cite this article:}

Ma Zefeng, Fang Lin. Seismic Design of a Out-of-code High-rise Library. Science Discovery. Vol. 8, No. 5, 2020, pp. 83-89. doi: $10.11648 /$ j.sd.20200805.12

Received: August 11, 2020; Accepted: September 8, 2020; Published: September 23, 2020

\begin{abstract}
In order to meet the requirements of architectural design form, a high-rise library project appeared many structural seismic designs, such as plane thin waist concave, large area floor missing, tower offset and torsion irregularity. In accordance with the principle of economy, safety and reliability, many calculation measures are adopted in the structural design, such as the comparative analysis of software SATWE and ETABS, the special calculation of floor stress level, the combining floor analysis of missing floors and the strengthening performance analysis under medium and large earthquake. At the same time, according to the structural transfinite items, the structural strengthening measures are taken, such as optimizing the structural layout, reducing the eccentricity, increasing the thickness of the floor at the weak position, and adding diagonal reinforcement in the stress concentration area. Using the above treatment, the structure can meet the fortification target under three levels.
\end{abstract}

Keywords: Wasp-waisted Plane, Floor Missing, Floor Slab Stress Analysis, Combining Floor Analysis

\section{某超限高层图书馆的抗震设计}

\section{马泽峰, 方林}

华东建筑设计研究院有限公司, 上海, 中国

邮箱

mzfmzf@sina.com（马泽峰）, lin_fang@arcplus.com.cn（方林)

摘要: 为满足建筑设计形式的要求, 某高层图书馆项目中出现平面细腰形凹进、楼板大面积缺失、塔楼偏置以及扭转 不规则等多项结构超限设计。遵循经济、安全、可靠的宗旨, 结构设计中采取了SATWE与ETABS对比分析、楼板应 力水平专项计算、楼板缺失楼层并层计算以及中、大震强化性能分析等多项计算措施。同时, 针对结构超限条目采取 了优化结构布置减少偏心、增加薄弱位置楼板厚度、应力集中区加配斜向钢筋等多项构造加强措施。经上述处理后的 结构可满足三水准下的设防目标。

关键词: 细腰形, 楼板缺失, 楼板应力分析, 并层计算

\section{1. 结构概况}

某市图书馆及综合服务中心等业务用房为新建工程, 地下 2 层, 地上 26 , 其中裙房 6 层, 塔楼结构高度 $99.55 \mathrm{~m}$, 裙房结构高度 $27.10 \mathrm{~m}$, 为钢筋混凝土框架-剪力墙结构。
建筑平面呈矩形形状, 地下室外围尺寸为 $119.55 \mathrm{~m} \times 159.00 \mathrm{~m}$; 裙房区段外围尺寸为 $79.20 \mathrm{~m} \times 96.90 \mathrm{~m}$; 塔楼标准层外围尺寸为 $50.40 \mathrm{~m} \times 50.40 \mathrm{~m}$ 。 
表1 地震作用相关参数表。

\begin{tabular}{llll}
\hline 设防类别 & 丙类 & 基本地震加速度 & $0.1 \mathrm{~g}$ \\
设防烈度 & 7度 & 设计地震分组 & 第一组 \\
场地类别 & III类 & 特征周期 & 0.45 秒 \\
阻尼比 & 0.05 & & \\
\hline
\end{tabular}

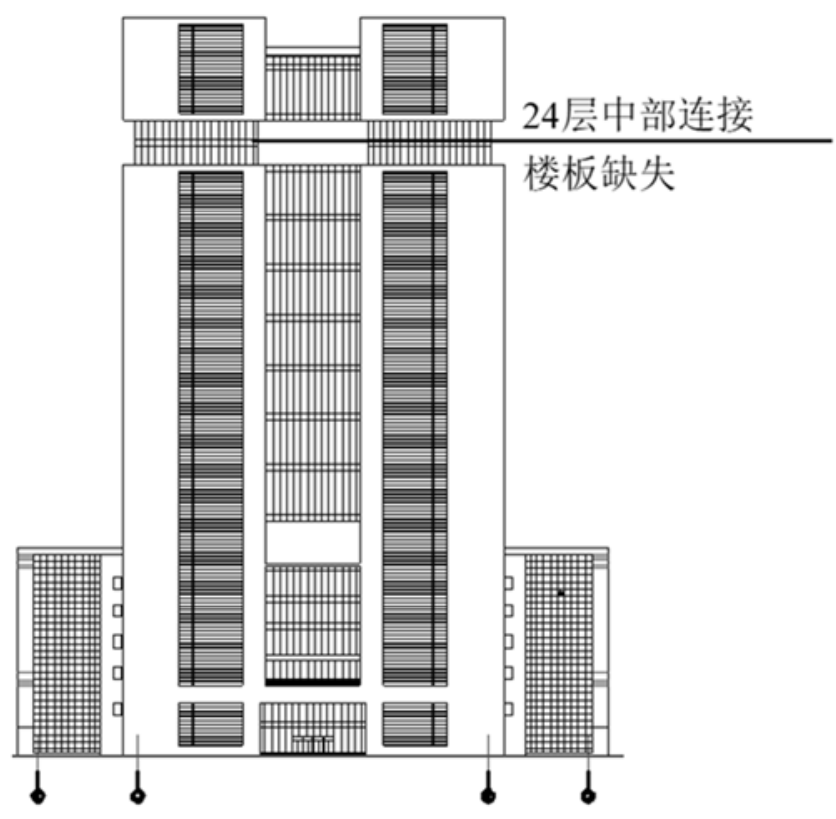

图1 建筑立面图。

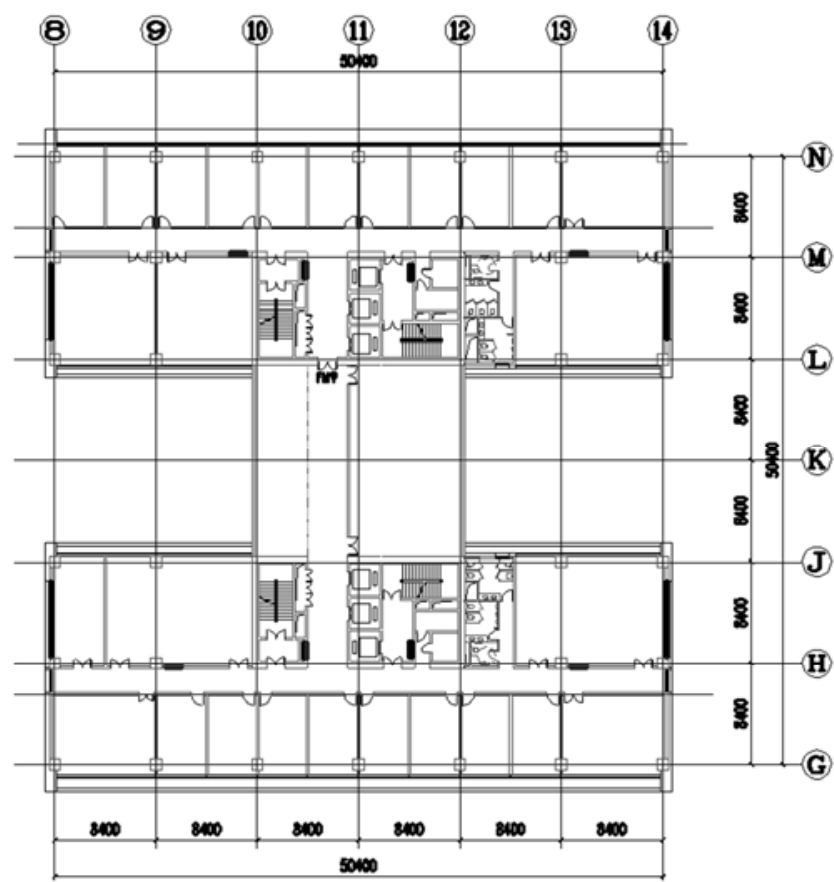

图2 标准层平面图。

工程存在以下平面不规则和坚向不规则的结构超限 内容:
1) 楼层的最大弹性水平位移大于该楼层两端弹性水 平位移平均值的1.2倍[1], 属扭转不规则;

2) 结构平面为细腰形, 结构平面凹进的一侧尺寸, 大于相应投影方向总尺寸的 $30 \%$, 属凹凸不规则 [2 4]（详见图2）;

3) 24 层楼板的尺寸和平面刚度急剧变化, 连接塔楼 楼板全部缺失，有效楼板宽度小于该层楼板典型 宽度的 $50 \%$ [5 8], 属楼板局部不连续（详见图 1);

4) 主楼位置明显偏置于大底盘[9 11], 属于平面不 规则;

\section{2. 结构总体指标对比计算结果}

为保证计算结果的准确性和可靠性, 采用中国建筑 科学研究院建筑工程软件研究所编制的PKPM系列软件 SATWE空间结构模型计算程序, 结构抗震计算按照扭转 耦联振型分解法进行, 考虑双向地震作用及偶然偏心影 响, 并采用ETABS (Ver9.5.0) 计算程序对SATWE结果 进行了对比和校核。计算中取 \pm 0.000 为嵌固端。取 24 个 振型进行了计算, 结构总重量约为 $1960676 \mathrm{kN}$ 。计算模型 详见图3, 详细的计算结果对比表见表2。

计算结果表明, 结构各项指标均满足规范要求。从 SATWE、ETABS反应谱计算结果来看，其计算结果相差 在 $5 \%$ 以内, 结构动力特性一致, 说明计算程序选择合适, 计算结果可靠。计算得到的周期、层间位移角及位移比 值等指标均在合理范围内, 并满足现行规范的要求。地 下一层与首层的侧向等效剪切刚度比值 $\mathrm{X}$ 向为5.820、Y 向为 5.675 , 满足 \pm 0.000 做为嵌固端的要求。各典型曲线 形状突变处均与结构特点相一致, 无明显薄弱部位。

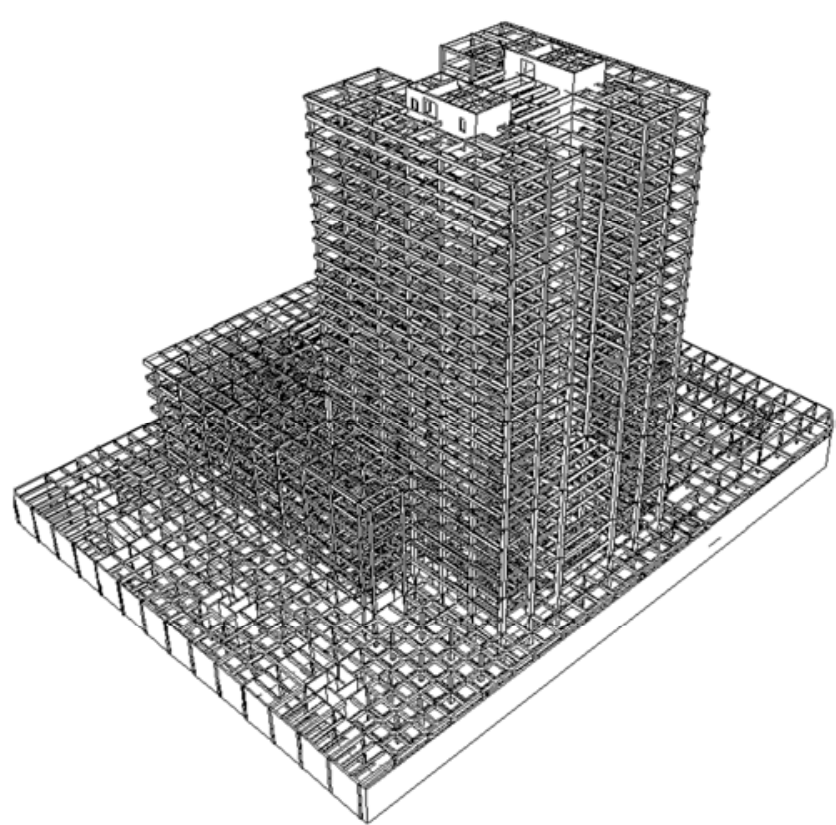

图3 计算模型示意图。 
表2 总体指标对比表。

\begin{tabular}{|c|c|c|c|c|}
\hline 分析软件名称 & & & SATWE & ETABS \\
\hline \multirow{4}{*}{ 周期 } & \multicolumn{2}{|l|}{$\mathrm{T} 1$ 平扭比例 $(\mathrm{X}+\mathrm{Y}, \mathrm{Z})$} & $2.9019(0.00+0.99,0.01)$ & 2.63075 \\
\hline & \multicolumn{2}{|l|}{$\mathrm{T} 2$ 平扭比例 $(\mathrm{X}+\mathrm{Y}, \mathrm{Z})$} & $2.1790(1.00+0.00,0.00)$ & 2.31712 \\
\hline & \multicolumn{2}{|l|}{$\mathrm{T} 3$ 平扭比例 $(\mathrm{X}+\mathrm{Y}, \mathrm{Z})$} & $2.0742(0.00+0.04,0.96)$ & 2.20415 \\
\hline & \multicolumn{2}{|l|}{$\mathrm{T} 3 / \mathrm{T} 1$} & 0.71 & 0.83 \\
\hline \multirow{4}{*}{ 地震作用 } & \multirow{2}{*}{ 最大层间位移角 } & X向 & 1/1667(18层) & $1 / 1576$ (25层) \\
\hline & & Y向 & 1/1156(20层) & $1 / 1241$ (20层) \\
\hline & \multirow{2}{*}{ 最大层间位移比 } & X向 & $1.24(2$ 层) & $1.204(1$ 层 $)$ \\
\hline & & Y向 & $1.37(1$ 层 $)$ & 1.39 (1层) \\
\hline \multirow{4}{*}{ 风荷载作用 } & \multirow{2}{*}{ 最大层间位移角 } & X向 & $1 / 4422$ (12层) & 1/4761(12层) \\
\hline & & Y向 & $1 / 2540$ (16层) & $1 / 3218$ (15层) \\
\hline & \multirow{2}{*}{ 最大层间位移比 } & X向 & $1.02(1$ 层 $)$ & 1.026 (2层) \\
\hline & & Y向 & 1.31 (5层) & $1.127(6$ 层) \\
\hline \multirow{2}{*}{\multicolumn{2}{|c|}{ 基底剪力(kN) }} & $\mathrm{V}_{\mathrm{x}}$ & 25861.52 & 25290 \\
\hline & & $V_{y}$ & 19610.28 & 20780 \\
\hline \multirow{2}{*}{\multicolumn{2}{|c|}{ 基底剪重比(\%) }} & $\mathrm{V}_{\mathrm{x}} / \mathrm{G}$ & $2.29 \%$ & $2.30 \%$ \\
\hline & & $\mathrm{V}_{\mathrm{y}} / \mathrm{G}$ & $1.74 \%$ & $1.86 \%$ \\
\hline \multirow{2}{*}{\multicolumn{2}{|c|}{ 地震作用倾覆弯矩(kN.m) }} & $\mathrm{M}_{\mathrm{x}}$ & 1069919 & 1011000 \\
\hline & & $\mathrm{M}_{\mathrm{y}}$ & 799339 & 814100 \\
\hline \multirow{2}{*}{\multicolumn{2}{|c|}{$\begin{array}{l}\text { 框架部分承担的倾覆力矩比例 } \\
\text { (1层) }\end{array}$}} & X向 & $22.88 \%$ & $31.2 \%$ \\
\hline & & Y向 & $23.97 \%$ & $31.8 \%$ \\
\hline
\end{tabular}

\section{3. 针对超限情况的分析措施}

\section{1. 针对细腰形及标准层楼板缺失超限的分析}

鉴于结构标准层普遍存在细腰形的凹进, 在裙房部 分 $(2 \sim 7)$ 层楼面板、屋面板存在若干开洞现象, 9层 还存在楼板缺失情况, 采用ETABS程序对结构中受力最 不利的裙房屋面层、9层及13层楼板进行了细化分析[12]。 目的在于确定楼板的实际受力状况, 应力水平及安全程 度, 找到薄弱部位, 为板内配筋提供依据, 从而确保水 平地震力的连续传递, 避免地震中因楼板局部振动或局 部破坏而产生结构安全性隐患 [13]。

ETABS程序中的壳单元是一个组合膜和板弯曲行为 的 3 或 4 节点单元, 可以用来模拟平面应力单元。壳单元 具有面内和面外的刚度, 在每个连接点激活所有 6 个自由 度, 使用一个变化的 4 至 8 个节点的数值积分公式来形成 壳的刚度。在单元的局部坐标系内, 应力和内力及弯矩 在2 乘2 的高斯积分点计算, 并向外插值到单元的节点。 对于单元应力或内力的大致误差, 可从连接同一节点不 同单元计算值的差异得到。程序中的壳单元模型见下图4。 壳单元内力 (也称为应力合力) 是在整个单元厚度积分 应力而得的力和弯矩。这些内力为:

膜方向内力:

$$
F_{11}=\int_{-t h / 2}^{t h / 2} \sigma_{11} d x_{3}, F_{22}=\int_{-t h / 2}^{t h / 2} \sigma_{22} d x_{3}
$$

膜剪切应力:

$$
F_{12}=\int_{-t h / 2}^{t h / 2} \sigma_{12} d x_{3}
$$

板弯曲弯矩:

$$
\begin{aligned}
& M_{11}=-\int_{-t h b / 2}^{t h b / 2} t \sigma_{11} d x_{3}, \\
& M_{22}=-\int_{-t h b / 2}^{t h b / 2} t \sigma_{22} d x_{3}
\end{aligned}
$$

板扭转弯矩:

$$
M_{12}=-\int_{-t h b / 2}^{t h b / 2} t \sigma_{12} d x_{3}
$$

板横向剪切内力:

$$
V_{13}=-\int_{-t h b / 2}^{t h b / 2} \sigma_{13} d x_{3}, V_{23}=-\int_{-t h b / 2}^{t h b / 2} \sigma_{23} d x_{3}
$$

正内力相应于在整个厚度上恒定的正应力状态。正 弯矩相应于一个沿厚度线性变化且在底部为正的应力状 态。因此:

$$
\begin{aligned}
& \sigma_{11}=\frac{F_{11}}{t}-\frac{12 M_{11}}{t_{b}^{3}} x_{3}, \\
& \sigma_{22}=\frac{F_{22}}{t}-\frac{12 M_{22}}{t_{b}^{3}} x_{3}, \\
& \sigma_{12}=\frac{F_{12}}{t}-\frac{12 M_{12}}{t_{b}^{3}} x_{3},
\end{aligned}
$$

$$
\sigma_{13}=\frac{V_{13}}{t_{b}}, \sigma_{23}=\frac{V_{23}}{t_{b}},
$$

$$
\sigma_{33}=0 \text { 。 }
$$





图4 壳单元节点连接和面定义。

计算中将7层、8层整层楼板定义为壳单元，弱连接部位单元进行细分。模型分网后的及程序计算后各工况下板应 力见下图5、6。

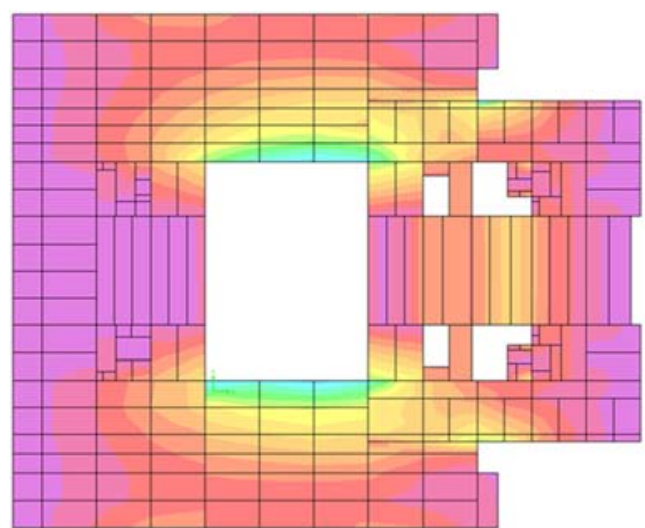

a) $\mathrm{S}_{\mathrm{MAX}}=0.656 \mathrm{Mpa}, \quad \mathrm{S1}_{\mathrm{MIN}}=-0.203 \mathrm{MPa}$

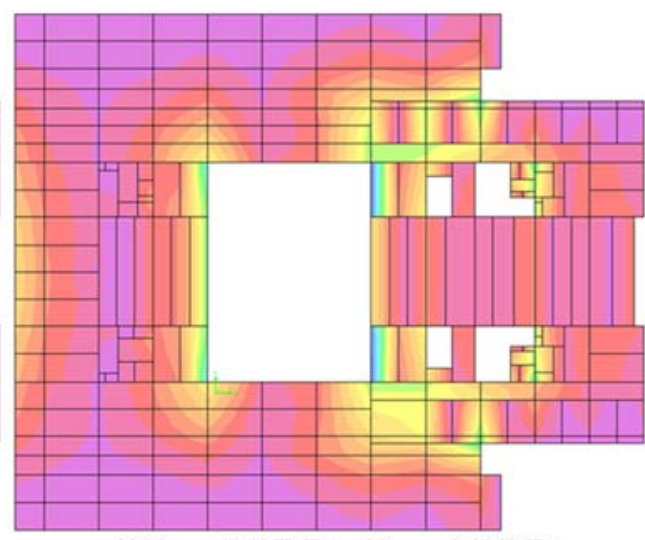

b) $2_{\mathrm{MAX}}=0.437 \mathrm{MPa}, \mathrm{S} 2_{\mathrm{MN}}=-0.122 \mathrm{MPa}$

图5 双向地震工况7层 (裙房屋面层) 楼板应力。
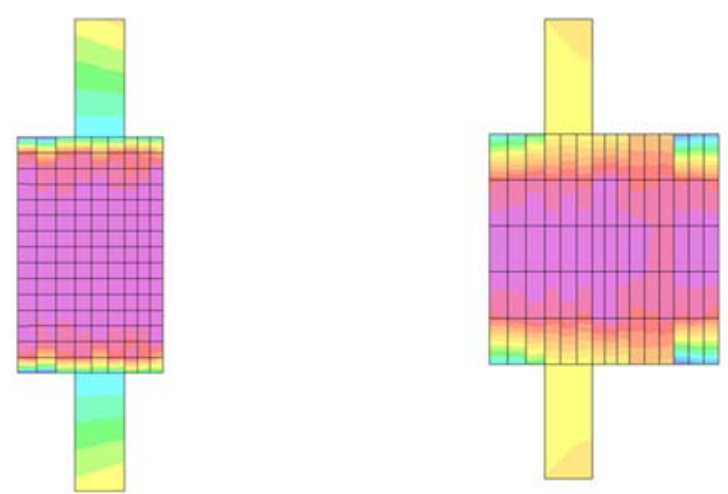

a) $S 1_{\mathrm{MAX}}=0.259 \mathrm{Mpa}$ （9层） b) $S 1_{\mathrm{MAX}}=0.301 \mathrm{Mpa}$ （13层）

图6 双向地震工况下楼板做大主应力。

从以上不同工况下板壳单元模型的计算结果可以得 出以下结论:

地震荷载作用下楼板在梁支座处负弯矩较大, 跨中 正弯矩较大, 符合板的受力特点。计算模型合理。

地震工况下楼板普遍处于较低的应力水平。
在框架梁边缘处, 开大洞的角部位置, 梁柱节点处 及板壳与剪力墙连接位置为板应力较为集中的区域, 楼 板配筋时应在这些部位予以适当加强。

地震工况中, 板内主应力在楼板与塔楼连接处应力 较大, 但整体分部均匀, 可以保证水平荷载的正常传递。 从上面的分析结果能看出, 虽然结构楼板在底部裙房层 有削弱, 但只要对应力集中的薄弱部位予以适当强化, 合理配筋, 此结构方案还是可以保证地震作用下结构的 整体性, 楼板可以确保水平面内、外刚度, 并给予坚向 构件合理约束。

\section{2. 针对 24 层塔楼连接楼板缺失超限的分析}

结构分析采用的是地上 26 层的模型, 考虑实际应用 中24层上、下两部分塔楼间并无梁或楼板拉结, 楼板缺 失情况严重, 为保证该层结构强度, 避免形成薄弱部位, 这里采用25层的计算模型, 合并 23 层和 24 层为一层, 层 高 $7.2 \mathrm{~m}$, 核对该模型楼层反应值, 并根据并层计算得到 的本层配筋校核 26 层模型的配筋结果 [14]。 
由计算结果可知, 采用并层计算模型时 23 层塔侧移 刚度与上一层相应塔侧移刚度 $70 \%$ 或上三层平均侧移刚 度 $80 \%$ 的比值中之较小者的比值分别为 X向 $0.9146, \mathrm{Y}$ 向 0.9336 , 该层按薄弱层处理, 楼层地震力放大 1.15 倍。但 由层间位移及层间位移角曲线来看, 并层计算后结构的
反映并未在该层发生明显的突变 (位移角X向 $1 / 1759, \mathrm{Y}$ 向 1/1280）, 各项指标均满足规范要求, 只要对该楼板 缺失层采用必要的结构措施, 楼层的局部变化不会影响 结构的整体性能。
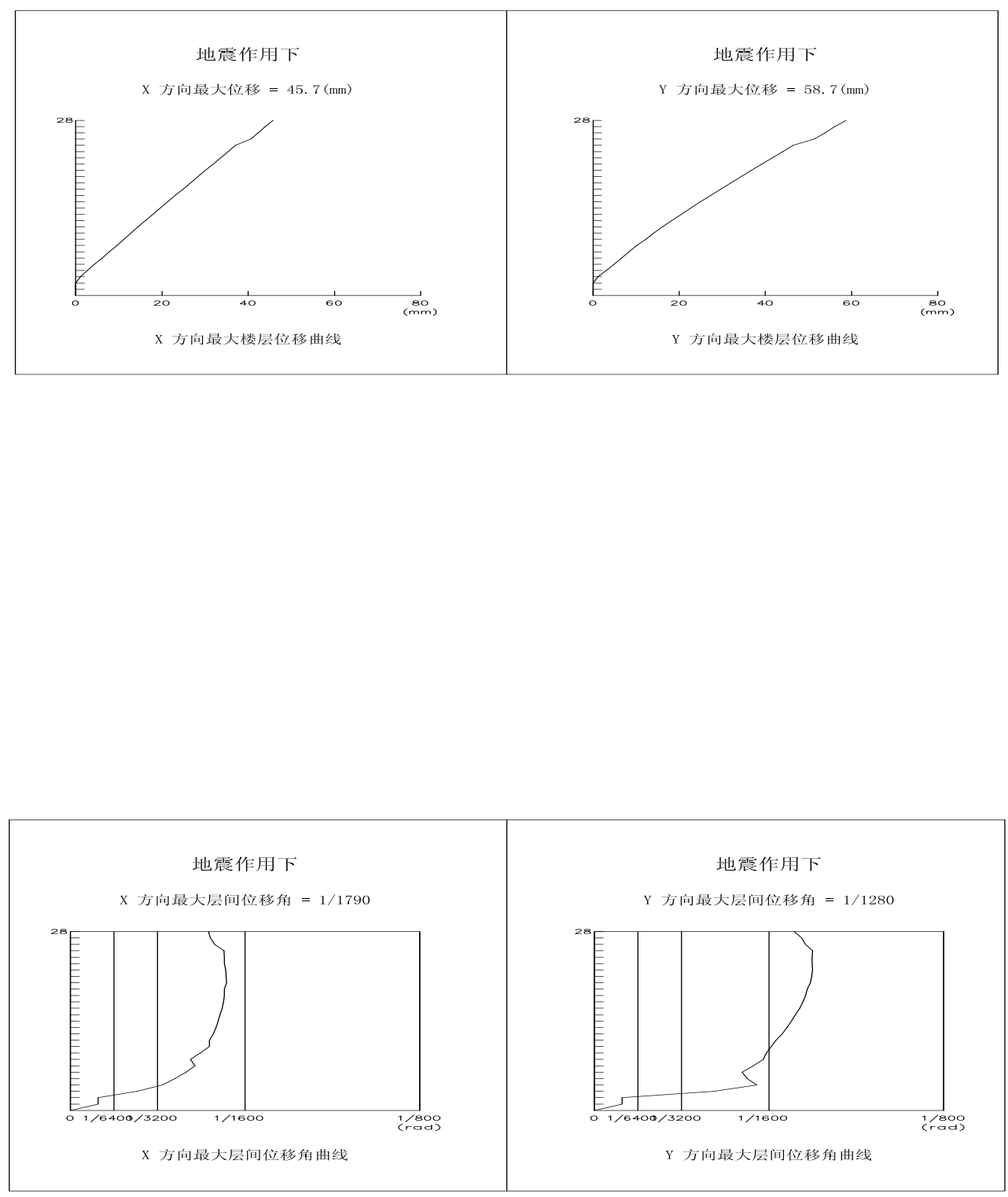

图7 并层计算模型楼层位移及层间位移角曲线。 


\section{4. 其它抗震措施}

抗震措施主要从优化结构体系、注重结构抗震概念 设计和构造、加强结构计算分析等方面, 采取对策和措 施确保该工程经济、安全、可靠。

\section{1. 针对超限结构采取的抗震措施}

针对扭转不规则及大底盘偏心布置措施: 优化结构布 置, 减少质量和刚度的偏心; 控制位移比不超过规范限 制的 1.4 ; 加强裙房角柱配筋（比计算值提高 $10 \%$ ），箍 筋全高加密; 结合建筑功能在裙房楼梯部位局部布置剪 力墙, 减少刚度偏心。针对细腰形不规则及楼板局部不 连续措施:加厚上下塔楼的连接板厚度, 采用双层双向配 筋, 适当提高配筋率; 增加板边缘框架梁截面, 纵筋通 常设置, 适当提高纵筋配筋率; 加强连接板洞口角部配 筋, 配置斜向钢筋; 加强楼板缺失层上下两层的框架柱 及剪力墙配筋。针对坚向不规则措施:加厚裙房屋面板厚 度，保证水平作用的有效传递；提高裙房楼板上下相邻 层的框架柱及剪力墙配筋; 控制楼层刚度比, 避免楼层 刚度突变[15]。

\section{2. 结构计算分析时采取措施}

多遇地震 (小震) 作用下采用两个不同力学模型的 三维空间分析软件进行内力和位移整体计算, 计算程序 为SATWE (最新版本) 和ETABS (Ver9.2.0), 对计算 结果进行详尽对比和分析, 保证分析结果的准确性。按 规范要求, 用SATWE进行弹性时程分析补充计算, 了解 结构在地震作用下的响应过程, 并籍此寻找结构薄弱部 位, 以便进行针对性的结构加强。时程分析的地震波采 用两条记录波和一条人工波。按现行规范考虑结构的扭 转效应, 使结构扭转周期与平动周期的比值、角部位移 与质心位移的比值均控制在规范要求的范围内。对于可 能出现的薄弱部位, 通过调整坚向构件的截面, 使相邻 层的坚向刚度比和楼层抗剪承载能力比在规范要求范围 内。塔楼部分连接板全楼采用弹性膜单元模拟, 考虑楼 板平面内真实刚度, 根据板内实际计算应力校核板配筋。

基本烈度（中震）作用下采用SATWE按7度中震进 行分析, 主楼底部加墙部位剪力墙的受剪承载力按中震 弹性, 拉弯、压弯承载力按中震不屈服设计。

罕遇地震（大震）作用下结构分析采用EPSA程序对 结构进行大震下作用的静力弹塑性推覆PUSHOVER 分析 [16], 分析大震下作用下结构的非线性地震反应过程, 确 保“大震不倒”性能目标。

\section{5. 总结}

综合上述论述结果, 从本超限高层建筑的结构设计 中可知:

1) 对于类似本工程的细腰形凹进平面或楼板缺失较 多的超限情况, 可通过细致的楼板应力分析, 找 到结构的薄弱连接部位, 从而进行有针对的加厚
楼板, 加强配筋等措施, 进而满足对楼板性能的 更高要求;

2) 当局部楼层楼板缺失面积大, 无法保证地震作用 连续传递的情况, 可通过并层计算, 通过对比并 层模型的整体指标, 采取相应放大地震作用或强 化构造措施等手段避免可能出现的薄弱层;

3) 大底盘偏置和扭转位移比超限的情况可从优化结 构布置, 减小整体偏心的角度进行;

4) 结构的概念设计尤为重要, 应从概念出发, 针对 薄弱部位或薄弱楼层采取加强措施。

采用上述分析设计和强化措施后的结构可满足规范 三水准的设防目标。

\section{致谢}

国家重点研发计划资助( 项目编号: 2017YFC0702900) (National Key R\&D Program of China (No.2017YFC0702900))。

\section{参考文献}

[1] 赵西安. 楼板变形对高层建筑结构内力与位移的影响及其 计算 [J].建筑结构.1982(6):1 9。

[2] 焦柯,赖鸿立,胡成恩, 吴桂广, 袁辉,彭子祥. 高层结构不规则 项判别条件的探讨及建议 [J].建筑结构. 2020(05). 50(10): 28 38。

[3] 黄涁辉.建筑平面不规则结构的动力特性与分析方法 [J]. 建 筑科技.2019(12).15 25。

[4] 全晓嵪,罗嘉骏.平面凹凸不规则高层建筑结构弹塑性时程 分析[J].建筑科学,2019(05).35(5):114 121。

[5] 何娟,刘乐乐,张先进. 框-剪结构楼板局部不连续的处理方法 对比分析 [J].建筑结构.2019(04).49(S1):250 254。

[6] 李传运,徐术陇,王健. 某坚向渐收框剪结构的设计 [J].建筑结 构.2018(10).48(19):31 35。

[7] 马泽峰. 地下室顶板开大洞对嵌固能力的影响[J].结构工程 师. 2012(02).28(2):19 24。

[8] 谭宇胜,刘岩.凹凸不规则平面高层建筑结构设计的思考 [J]. 广东土木与建筑.2020(05).27(5):37 39。

[9] 许铭,陈德良,徐强,㫿才伟.武汉华侨城某超高层建筑抗震性 能设计 $[\mathrm{J}]$.建筑结构.2019(05).49(9):33 38。

[10] 张晓旭,郭天焓,刘巍,何敬天.西安万科大明宫住宅项目结构 设计与分析[J].建筑结构.2019.49(S1):131 134。

[11] 中华人民共和国建设部. JGJ 3-2010 高层建筑混凝土结构 技术规程[S]. 北京: 中国建筑工业出版社,2010。

[12] 法扎德.奈姆.抗震设计手册 $[M]$, 中国建筑出版社,2008。 
[13] 黄婷婷.上海枫溪萃园平面凹凸不规则建筑超限结构分析 [J].上海建设科技.2020(03):37 39。

[14] 谭宇胜,刘岩.凹凸不规则平面高层建筑结构设计的思考 [J]. 广东土木与建筑.2020(05).27(5):37 39。
[15] 胡长青.平面不规则高层建筑结构设计分析 [J].工程建设与 设计.2019(07).12 14。

[16] 刘慧征. 某不规则超限高层设计研究 [D]. 石家庄铁道大 学.2019。 